\title{
FIB/SEM Processing of Biological Samples
}

Annalena Wolff ${ }^{1}$, Yinghong Zhou ${ }^{2,4}$, Jinying Lin $^{3,4}$, Yong Y Peng ${ }^{5}$, John A.M. Ramshaw ${ }^{5,6}$ and Yin $\mathrm{Xiao}^{2.4}$

1. Central Analytical Research Facility, Institute for Future Environments, Queensland University of Technology (QUT), Brisbane, Australia.

2. Institute of Health and Biomedical Innovation, Queensland University of Technology (QUT), Brisbane, Australia.

3. Department of Implantology, Xiamen Stomatological Research Institute, Xiamen Stomatological Hospital, Fujan, China.

4. The Australia-China Centre for Tissue Engineering and Regenerative Medicine (ACCTERM), Queensland University of Technology, Brisbane, Australia.

5. CSIRO Manufacturing, Bayview Avenue, Clayton, Australia.

6. Department of Surgery, St. Vincent's Hospital, University of Melbourne, Australia.

Combining a Focused Ion Beam (FIB) with a Scanning Electron Microscope (SEM) provides a unique capability for revealing a sample's subsurface structures, preparing transmission electron microscopy (TEM)-lamellas as well as for reconstructing 3D sample models at precisely selected points with nanometre resolution. The FIB/SEM has become the "go to" tool in the materials sciences and semiconductor industry, however, it is not yet fully established in the biological sciences [1-5]. This is predominantly due to the heat-induced damage from the ion beam when processing soft materials including biological samples. Appropriate FIB parameters for processing non-embedded biological samples and soft materials, which limit artefacts and sample damage are not yet well understood.

This study focuses on the underlying ion-solid interactions and the effect of processing parameters on heating induced by ion beams. The work presented here deals with gallium ion solid interactions, however the broader results are applicable to any type of FIB including the helium ion microscope (HIM) and plasma FIBs. The interactions of gallium ions in skin were simulated using Monte Carlo methods. The program SRIM [6] was used to obtain theoretical results which permit estimation of the ion beam induced temperature increases, using the physical principles of Fourier's law of conductive heat transfer. The model suggests that the ion beam induced increase in temperature can be reduced by:

1. Reducing the local dose rate (smaller aperture/ion beam current)

2. Reducing the local dose (smaller aperture/ion beam current, introducing beam blur, reducing the beam overlap)

The technique was tested on collagen, a soft biological material which is commonly used in biomedical applications. Collagen was chosen as a suitable test sample as it loses its fibrillary structure when denaturated by heat, permitting damage to easily be recognized. Cross-sections and TEM lamellas were prepared from non-embedded collagen with conventional FIB processing parameters (see Figure 1 left) as well as heat reducing FIB parameters (see Figure 1 right).

The results also show that heat damage can be prevented by reducing the local dose rate and area underneath the ion beam. A TEM comparison of a microtome prepared lamella and a FIB prepared lamella (using heat reducing parameters) shows that the fibrillar structures can be maintained, and heat 
damage avoided. The approach described here can be used to determine suitable parameters for other soft materials [7].

\section{References:}

[1] Drobne et al, Microscopy Research and Technique 70 (2007), p. 895.

[2] Earl et al, Journal of Microscopy 240 (2010), p. 1.

[3] Schneider et al, Bone 49 (2011), p. 304.

[4] Stokes et al, Journal of Physics: Conference Series 26 (2006), p. 50.

[5] Bandara et al, ACS Applied Materials \& Interfaces 2017 9(8) (2017), p. 6746.

[6] Ziegler et al, www.srim.org.

[7] The authors acknowledge scientific and technical assistance of Peter Hines, Jamie Riches, Rachel Hancock, and Ning Liu and the facilities at the Australian Microscopy \& Microanalysis Research Facility (AMMRF) at the Central Analytical Research Facility (CARF), Queensland University of Technology, Brisbane, Australia.

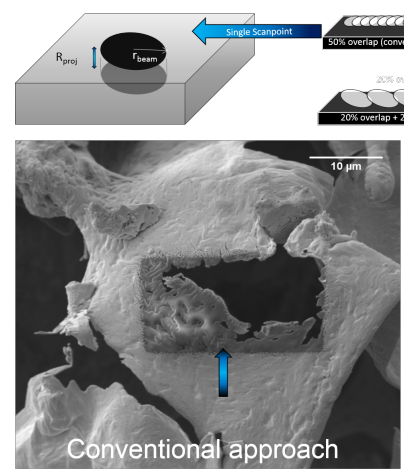

Figure 1. SEM images showing FIB prepared crosssections in collagen with conventional parameters (left) and heat reducing parameters (left). The difference between the parameter sets is illustrated in the top part of the image. 